\section{Erdnüsse für Mäusemütter - Anaphylaxieschutz für Mäusekinder}

\begin{abstract}
Schwangeren und Stillenden wurde lange Zeit nahegelegt, auf Erdnüsse zu verzichten. Erst seit 2009 wird in den offiziellen Leitlinien von jeglicher restriktiven Diät mit dem Ziel der Allergieprävention abgeraten - eine Empfehlung, die durch aktuelle Daten bekräftigt wird.
\end{abstract}

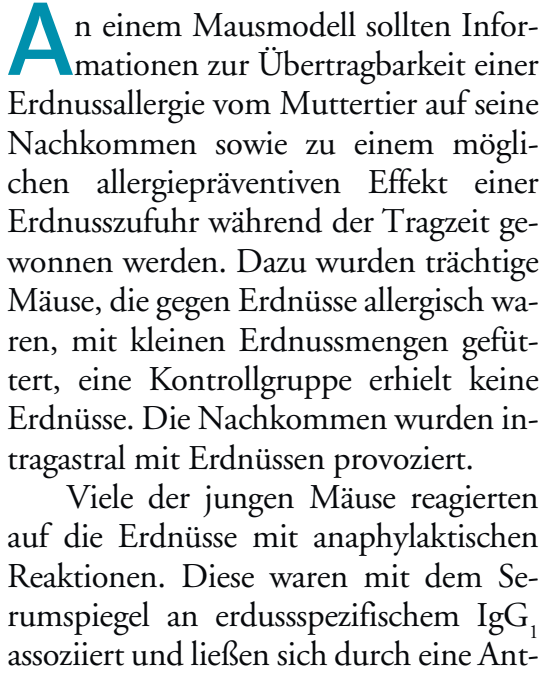

agonisierung des plättchenaktivierenden Faktors verhindern. Waren die Mäusemütter mit kleinen Erdnussmengen gefüttert worden, entwickelten ihre Nachkommen mit $28 \%$ aber signifikant weniger allergische Reaktionen bei der Erdnuss-Erstprovokation als die Kontrollgruppe mit $80 \%$. Die so geschützten

Tiere hatten erhöhte Spiegel an spezifischem $\operatorname{IgG}_{2 \mathrm{a}}$ und zeigten eine verminderte mitogenstimulierte Zytokinproduktion in Splenozyten verglichen mit den NachkommenvonMuttertieren, die keine Erdnüsse erhalten hatten.
Fazit: Die Übertragung einer Erdnussallergie von der Mutter auf ihr Kind erfolgt möglicherweise über erdnussspezifisches $\mathrm{IgG}_{1}$. Moderater Erdnussverzehr während der Schwangerschaft reduziert das Risiko einer Erdnussanaphylaxie der Nachkommen. Die Schutzwirkung wird offenbar durch vermehrt gebildetes spezifisches $\mathrm{IgG}_{2}$ vermittelt. Ob sich diese im Tierversuch gefundenen Ergebnisse auf den Menschen übertragen lassen, ist noch offen.

López-Expósito l et al. Maternal peanut exposure during pregnancy and lactation reduces peanut allergy risk in offspring. J Allergy Clin Immunol 2009; 124: 1039-46

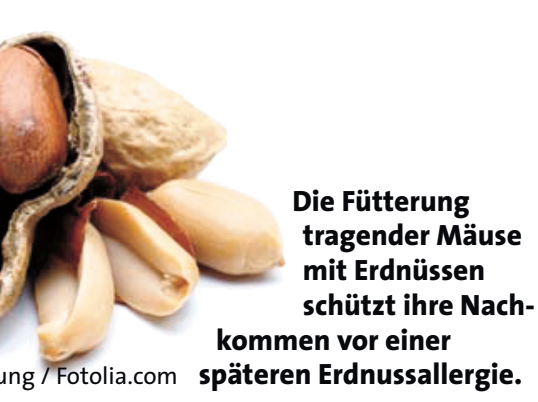

\section{Allergieprävention durch Synbiotika?}

\section{Eine frühe Modulation der Immunantwort über die Ernährung könnte Kinder vor der Entwicklung von Allergien, beispielsweise gegen Kuhmilch, schützen. An jungen Mäusen wurde jetzt der Einfluss einer kombinierten Supplementation mit Pro- und Prebiotika auf die Sensibilisierung gegen Molke untersucht.}

\footnotetext{
E s gibt eine Reihe von Oligosacchac riden, die für den Menschen unverdaulich sind, aber immunmodulatorisch wirkenden Darmbakterien als Nährstoffe dienen. Die simultane Gabe von solchen Oligosacchariden (Prebiotika) und Bakterien (Probiotika) ist möglicherweise ein sinnvoller allergiepräventiver Ansatz. Eine solche Kombination nennt man Synbiotikum.

In einer niederländischen Studie wurde die Schutzwirkung von Probiotika, Prebiotika und Synbiotika im Hinblick auf eine Sensibilisierung gegen Molke an Mäusen untersucht. Dazu wurde den Tieren folgende Diät verabreicht: eine Prebiotika-Mixtur von kurzkettigen Galakto-Oligosacchariden und
}

langkettigen Fructo-Oligosacchariden (Immunofortis) oder die probiotisch aktive Bakterienspezies Bifidobacterium breve (Stamm M-16V) oder eine synbiotische Mischung aus Pre- und Probiotikum. Zwei Wochen nach dem Beginn der Diät erfolgte die erste Sensibilisierung. Die allergische Reaktion auf eine intradermale Provokation am Ohr wurde anhand der Hautreaktion und eines Anaphylaxie-Scores gemessen, zusätzlich wurden Blutproben genommen.

Mäuse, an die das Synbiotikum verfüttert worden war, zeigten eine deutlich herabgesetzte Hautreaktion und geringere Anaphylaxie-Scores im Vergleich zu Kontrollmäusen $(p<0,01)$. Das Pre- und das Probiotikum alleine verminderten die allergische Hautreaktion dagegen weitaus weniger und beeinflussten den Anaphylaxie-Score nicht. Die molkespezifischen IgE- und $\mathrm{IgG}_{1}$-Antworten veränderten sich in keiner Untersuchungsgruppe. Die Spiegel der $\operatorname{IgG}_{2 \mathrm{a}}$-Antikörper waren jedoch in allen Interventionsgruppen höher als bei den Kontrollen $(p<0,05)$. Dies lässt auf eine verbesserte TH1- oder regulatorische T-Zell-Antwort schließen und kann als allergiepräventiver Effekt gedeutet werden.

Fazit: Die orale Zufuhr einer Kombination eines Oligosaccharid-haltigen Prebiotikums und eines Bifidobakterienstamms als Probiotikum vermindert im Mausmodell allergische Reaktionen nach Sensibilisierung gegen Molke. Möglicherweise lässt sich mit einer solchen synbiotischen Mischung die Entwicklung einer Nahrungsmittelallergie auch beim Menschen verhindern.

Schouten B et al. Cow milk allergy symptoms are reduced in mice fed dietary synbiotics during oral sensitization with whey. J Nutr 2009; 139: 1398-1403 